\title{
Comparison of botulinum toxins for treatment of movement disorders: real-world utilization and cost analysis in a national Medicare population
}

Rashid Kazerooni, PharmD, MS, BCPS, and Jonathan H Watanabe, PharmD, MS, PhD, BCGP

\section{What is already known about this subject}

- Medicare Part B drug spend on the botulinum toxin drug class has increased from \$236 million in 2014 to $\$ 382$ million in 2018.

- Potential for Centers for Medicare \& Medicaid Services cost savings exists given presence of therapeutically equivalent less-expensive botulinum toxin agents.

\section{ABSTRACT \\ BACKGROUND: The Centers for Medicare \& Medicaid Services (CMS) is the single largest payer for health care in the United States and the largest payer by spending globally. Medicare Part B, with more than 50 million beneficiaries, currently has no broad mecha- nisms in place for promoting cost-effective care of injectable drugs.}

OBJECTIVE: To conduct a real-world utilization and cost analysis comparing botulinum toxins in movement disorders.

METHODS: The 2017 Medicare Provider Utilization and Payment Data: Physician and Other Supplier dataset from CMS was used for this claims level analysis. Neurologists,

\author{
What this study adds \\ - This study, to the authors' knowledge, \\ is the largest provider (891 physicians \\ who inject predominantly for movement \\ disorders) and patient population \\ (national cohort of 34 million Medicare \\ lives) studied to date in terms of real- \\ world botulinum toxin use and costs. \\ - IncobotulinumtoxinA was shown to be \\ less costly for both payer and patients, \\ with lower wastage and a similar \\ dosing ratio when compared with \\ onabotulinumtoxinA. \\ - Increasing incobotulinumtoxinA use \\ in the movement disorder space to \\ attain an overall $20 \%$ botulinum toxin \\ market share was estimated to reduce \\ Medicare Part B spending by $\$ 32.9$ \\ million over a 3-year period versus \\ current practice.
}

ophthalmologists, or physiatrists who injected predominantly for movement disorders (defined as blepharospasm, cervical dystonia, sialorrhea, and/or spasticity) were included along with their patients. Botulinum toxins with FDA indications spanning these 3 specialties were included.

RESULTS: A total of 891 physicians (406 ophthalmologists, 338 neurologists, and 147 physiatrists) along with their 29,954 botulinum toxin $(27,441$ onabotulinumtoxinA and 2,513 incobotulinumtoxinA) patients were included in the analysis. The average total drug cost per patient per year (PPPY) was significantly lower for incobotulinumtoxinA versus onabotulinumtoxinA ( $\$ 2,099$ vs. $\$ 3,115 ; P<0.001)$, for an average savings of $32.6 \%$. Annual average out-of-pocket

\section{Author affiliations \\ Rashid Kazerooni, PharmD, MS, BCPS, Merz Pharmaceuticals, Raleigh, NC, and Jonathan H Watanabe, PharmD, MS, PhD, BCGP, School of Pharmacy and Pharmaceutical Sciences, University of California, Irvine.}

\section{AUTHOR CORRESPONDENCE:}

Rashid Kazerooni,

rashid.kazerooni@merz.com

J Manag Care Spec Pharm 2021;27(4):478-87

Copyright $\odot 2021$, Academy of Managed Care Pharmacy. All rights reserved.

costs were also significantly less expensive for incobotulinumtoxinA versus onabotulinumtoxinA ( $\$ 486$ vs. $\$ 719 ; P<0.001$ ), for an average savings of $32.4 \%$. Across 74,346 total injection visits, there was no significant difference in dosing between the agents, with an average dosing ratio of 0.94 incobotulinumtoxinA to 1.0 onabotulinumtoxinA. Injections PPPY were 2.42 for onabotulinumtoxinA and 2.29 for incobotulinumtoxinA. Average reported wastage was $64 \%$ higher for onabotulinumtoxinA than it was for incobotulinumtoxinA. A budget impact analysis estimated that increasing incobotulinumtoxinA use in the movement disorder space to attain an overall $20 \%$ botulinum toxin market share would save Medicare $\$ 32.9$ million over a 3-year period versus current use. 
CONCLUSIONS: IncobotulinumtoxinA was shown to be a less costly alternative than onabotulinumtoxinA with similar dosing in realworld practice in this large national Medicare population. Policies to increase use of agents that promote cost-effective evidencebased care should be further explored and implemented for this fundamental federal payer.

Medicare Parts B and D total drug spending has increased dramatically over the past several years, from $\$ 140$ billion in 2014 to \$201 billion in 2018, ${ }^{1,2}$ Medicare Part B, which covers infusible and injectable in-clinic drugs, makes up more than $15 \%$ of all Medicare drug spending, even before accounting for Medicare Advantage beneficiary data. ${ }^{1,2}$ However, unlike Medicare Part D, which is typically managed by pharmacy benefit management organizations, Medicare Part B has no such mechanism in place for incentivizing cost-effective care. Current Medicare Part B reimbursement policies may effectively contribute to care that runs counter to value-based treatment by reimbursing on the manufacturer-derived average sales price (ASP) plus $4.3 \%{ }^{3}$

The Centers for Medicare \& Medicaid Services (CMS) is the single largest payer for health care in the United States. ${ }^{4}$ Medicare enrollees represent the largest patient population with more than 60 million Medicare beneficiaries. ${ }^{5}$ Although many patients have supplemental insurance along with their Medicare coverage, it's estimated that $19 \%$ of traditional Medicare beneficiaries do not. ${ }^{5}$ Inefficient use of prescription drug resources can lead to increased out-of-pocket (OOP) costs for patients in addition to overall health system costs as a whole. Decision makers would benefit from an understanding of the annual spending estimates, waste medication costs, and potential reductions in spend if alternative botulinum toxins (BoNT) were prescribed. The annual Medicare Part B spending on BoNT is $\$ 382$ million with onabotulinumtoxinA (Botox, Allergan) representing $91.2 \%$ of this spend. ${ }^{1}$ A significant portion of BoNT total spend was attributable to waste with \$24 million (6.3\% of drug-class spend) paid for discarded units in 2017, outpacing rates of wastage payments on other Medicare Part B drugs (2.1\%). ${ }^{6}$ IncobotulinumtoxinA (Xeomin, Merz Pharmaceuticals) has demonstrated similar efficacy in multiple head-to-head randomized double-blind studies at a lower cost. ${ }^{1,7,8}$ Additionally, availability of incobotulinumtoxinA in smaller vial sizes may translate to reduced waste and consequently decreased spending on discarded medications. ${ }^{9,10}$

The goals of this nationally representative analysis were to determine these CMS costs, OOP costs and budget impacts among BoNT movement disorder agents while factoring in discarded units from the payer perspective.

\section{Methods}

\section{PROVIDER-LEVEL ANALYSIS}

The Medicare Provider Utilization and Payment Data: Physician and Other Supplier dataset from CMS was used for the claims level analysis. ${ }^{11}$ The most recent year of publicly available data from 2017 was used. Inclusion criteria included the following: (a) physician specialty was neurology, ophthalmology, or physical medicine and rehabilitation; (b) prescribers were estimated to have at least $50 \%$ of their BoNT patients being treated for movement disorders; and (c) physicians had reported claims for a BoNT agent with U.S. Food and Drug Administration (FDA) indications spanning all 3 aforementioned specialties (onabotulinumtoxinA and/or incobotulinumtoxinA).

Movement disorder indications were defined as treatment of any of the following: blepharospasm, cervical dystonia, spasticity, or sialorrhea. This subgroup along with the specialty restrictions was chosen to ensure that similar physicians and patient populations were compared between the 2 drugs, as these constitute the majority of overlapping FDA indications between these products, as well as the types of physicians that inject for these indications. The number of unique patients and unique visits for each respective drug was identified by the following J codes, which are aggregated at the provider level under the Healthcare Common Procedure Coding System variable in the aforementioned dataset: J0585 (injection, onabotulinumtoxinA, 1 unit) and J0588 (injection, incobotulinumtoxinA, 1 unit).

Provider, patient, BoNT, indication, and population mix were determined by pulling the following Current Procedural Terminology, 4th edition (CPT-4) codes, which are aggregated at the provider level under the aforementioned dataset: 64611 (injection of chemical for destruction of salivary glands on both sides of the mouth); 64612 (injection of chemical for destruction of nerve muscles on 1 side of face); 64616 (injection of chemical for destruction of nerve muscles on 1 side of neck excluding voice box accessed through the skin); 64642 (injection of chemical for destruction of nerve muscles on arm or leg, 1-4 muscles); or 64644 (injection of chemical for destruction of nerve muscles on arm or leg, 5 or more muscles). The sum of unique beneficiaries treated for these CPT codes (bene_unique_cnt variable) were then divided by the unique number of BoNT patients for each respective provider to get an estimated 


\section{FIGURE 1 Provider Inclusion Flowchart}

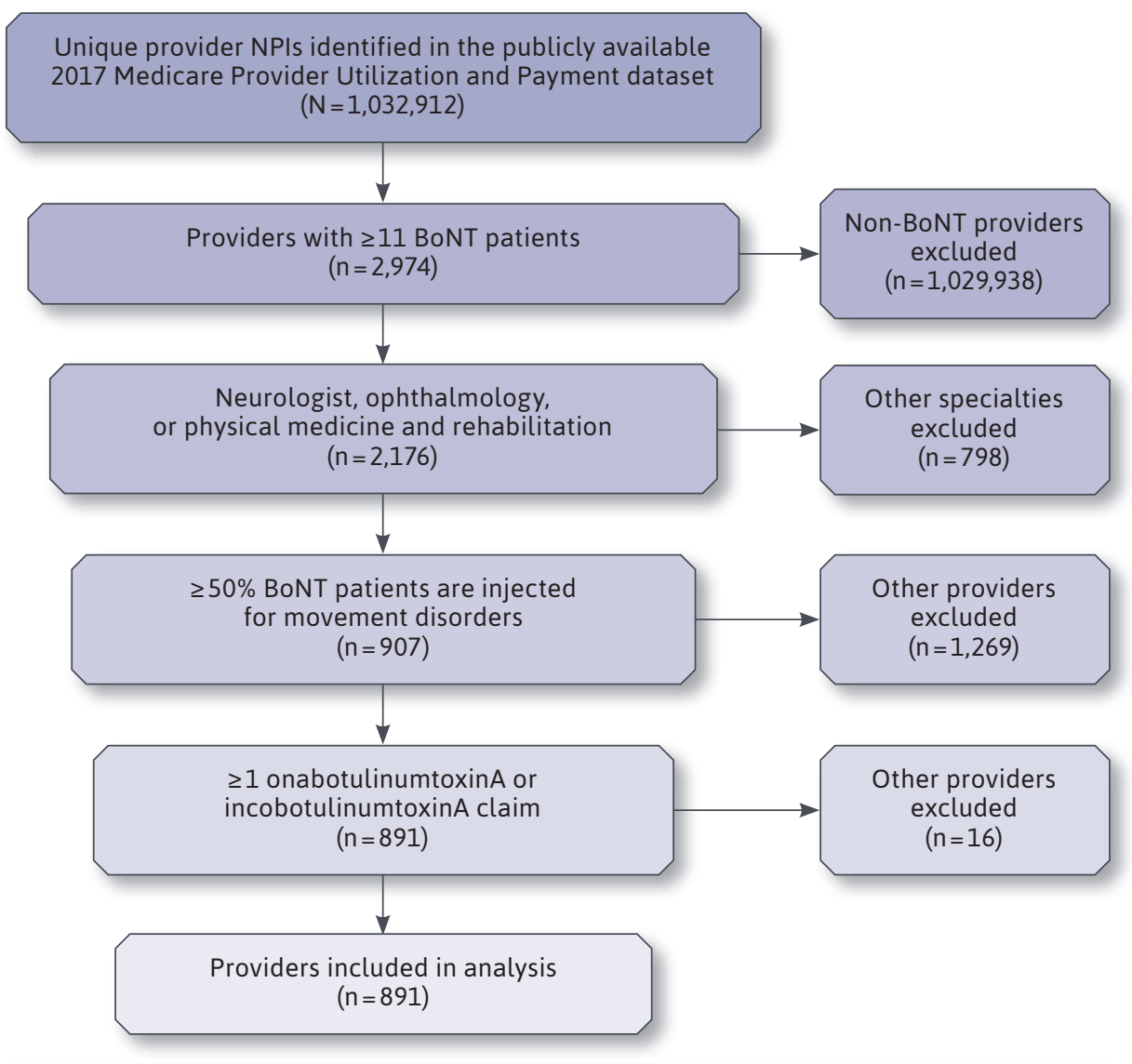

BoNT = botulinum toxin; $\mathrm{NPI}=$ National Provider Identifier

percentage of the BoNT patients who are being treated for movement disorders for each given provider.

Total costs were calculated by multiplying Medicare-allowed amount per unit (average_Medicare_allowed_ amt variable) for each respective drug times the number of units prescribed. Medicare costs were calculated by multiplying Medicare-paid amount per unit (average_Medicare_payment_amt variable) for each respective drug times the number of units prescribed. OOP cost was calculated by subtracting Medicare costs from the total costs, for each drug, respectively. Costs were then broken down by specialty, because each one has a specific mix of indications for which they prescribe, and this allows for a similar comparison between drugs.

The Medicare provider-level dataset does not report wastage; thus assumptions were made to account for this. Average wastage is reported by Medicare for each drug, respectively. ${ }^{6}$ However, wastage is only reported at the aggregate level and is not broken down by specialty, indication, etc. Therefore, for the majority of circumstances, this average wastage rate for each respective drug from the most recent reported Medicare wastage rates were added on to the costs for each drug.

A different methodology was used to account for wastage for onabotulinumtoxinA use in ophthalmology. It is well documented in the literature that the average dose for blepharospasm treatment is typically substantially less than 100 units, which is onabotulinumtoxinA's smallest vial size. IncobotulinumtoxinA is formulated in a 50-unit vial size. Thus the following was assumed for estimating wastage/costs for ophthalmology onabotulinumtoxinA injections: (a) for physicians who averaged $\leq 100$ units per visit, it was assumed that the provider used one 100-unit vial per patient per visit and (b) for physicians who averaged $>100$ units per patient per visit, it was assumed that the provider used one 100-unit vial for half of their patient visits and two 100-unit vials for the other half.

The primary outcome was the average total drug cost per patient per year (PPPY). Continuous variables such as dose, number of injections, and costs PPPY were assessed statistically by attaining means and standard deviations at the provider level. Descriptive and demographic variables are also presented when available. Cost and utilization outcome measures were compared within specialty as well as on an overall total basis, grouped by drug. For total costs and doses among all specialties combined, weighted averages were used to give equal onethird weighting to each specialty to account for any differences in specialty composition for the respective drugs. Market share for incobotulinumtoxinA versus onabotulinumtoxinA was calculated by number of 100-unit vial equivalents used respectively for each product. The data files from the CMS website were downloaded into R-studio version 3.6.2 (R-Tools 


\begin{tabular}{|c|c|c|}
\hline & $\mathbf{n}$ & $\%$ \\
\hline Physicians & 891 & - \\
\hline Gender, male & 685 & 76.9 \\
\hline Total BoNT patients & 31,222 & - \\
\hline $\begin{array}{l}\text { BoNT patients being treated for } \\
\text { movement disorder }\end{array}$ & 27,407 & 87.8 \\
\hline Average BoNT patients per provider & 35 & - \\
\hline Physicians with $100+$ BoNT patients & 49 & 5.5 \\
\hline \multicolumn{3}{|l|}{ Specialty } \\
\hline Ophthalmology & 406 & 45.6 \\
\hline Neurology & 338 & 37.9 \\
\hline PM\&R & 147 & 16.5 \\
\hline \multicolumn{3}{|l|}{ Drug choice } \\
\hline OnabotulinumtoxinA only & 779 & 87.4 \\
\hline Both drugs & 58 & 6.5 \\
\hline IncobotulinumtoxinA only & 54 & 6.1 \\
\hline \multicolumn{3}{|l|}{ Practice location } \\
\hline California & 94 & 10.5 \\
\hline Florida & 75 & 8.4 \\
\hline New York & 70 & 7.9 \\
\hline Texas & 57 & 6.4 \\
\hline Pennsylvania & 49 & 5.5 \\
\hline Ohio & 41 & 4.6 \\
\hline Illinois & 38 & 4.3 \\
\hline All other & 467 & 52.4 \\
\hline
\end{tabular}

BoNT = botulinum toxin; $P M \& R=$ physical medicine and rehabilitation.

Technology, Boston, MA) for the creation of a database with the relevant information for this analysis, as well as the analyses themselves. Where appropriate, a chi-square test for discrete data and Student's t-test for continuous measures were used to assess statistical significance. Significance level was set a priori at $\mathrm{P}<0.05$.

\section{BUDGET IMPACT ANALYSIS}

The CMS Part B drug spending dashboard was used. ${ }^{1}$ The analysis was conducted on the latest available data using CMS calendar year 2018 from which to project forward. These data were used to project a 3-year time horizon for years 2021-2023. A policy promoting cost-effective BoNT-A therapy was assumed to be implemented with an assumed market share rate of $20 \%$ for incobotulinumtoxinA within the entire BoNT drug class. A sensitivity analysis was also conducted ranging market shares for incobotulinumtoxinA from $10 \%$ to $30 \%$. The base-case market share assumption, as well as the sensitivity-analysis range, fall within a plausible range of attainable market share for incobotulinumtoxinA generally using only on-label indications, though lowerlimb spasticity was included due to combined spasticity data and is not itself an FDA-approved indication for incobotulinumtoxinA. This is supported by a 2019 analysis of 15 million commercially insured lives that showed that per member per month BoNT costs for blepharospasm, cervical dystonia, and spasticity accounted for $33.3 \%$ of total BoNT spend, even without accounting for sialorrhea. ${ }^{12}$ Market share for incobotulinumtoxinA versus onabotulinumtoxinA was used for this analysis and was calculated by number of 100-unit vial equivalents used respectively for each product. ASP was kept constant and not adjusted for inflation. The most recent Medicare Part B ASP rates from 2018 were carried forward, as ASP growth for the drug class over the past 5 years has been minimal and future BoNT-A approvals may limit price increases. A conservative $6 \%$ growth rate in drug-class use was assumed, which is significantly lower than the 2012-2018 average growth rate of over 13\%. As incobotulinumtoxinA is also available therapeutically in 50 -unit vials, an additional 10\% savings beyond ASP savings was assumed in incobotulinumtoxinA patients secondary to reduced wastage. AbobotulinumtoxinA and rimabotulinumtoxinB were assumed to stay constant in Medicare spend over the study time frame.

\section{Results}

\section{PROVIDER-LEVEL ANALYSIS}

There were a total of $1,032,912$ unique providers in the Medicare database for 2017, of which 2,974 had claims for BoNT. Of these providers, a total of 891 physicians met inclusion criteria and were included in the analysis, along with data from their 29,954 combined unique onabotulinumtoxinA and incobotulinumtoxinA movement disorder patients (Figure 1). These 2 drugs accounted for $95.9 \%$ of all 31,222 unique BoNT patients from these physicians (87.9\% onabotulinumtoxinA and $8.0 \%$ incobotulinumtoxinA). The top $10 \%$ of prescribers accounted for $42.2 \%$ of total BoNT costs (Table 1 ).

\section{COST AND UTILIZATION OUTCOMES}

Total cost PPPY was significantly less for incobotulinumtoxinA versus onabotulinumtoxinA across all specialties (all with $\mathrm{P}<0.001)$ as follows: neurology $(\$ 2,584$ vs. \$3,331), ophthalmology (\$884 vs. \$1,550), physical medicine and rehabilitation (\$2,829 vs. \$4,465), and overall (\$2,099 vs. $\$ 3,115$; Figure 2). Medicare cost PPPY was significantly less 


\section{FIGURE 2 Annual Costs per Patient}

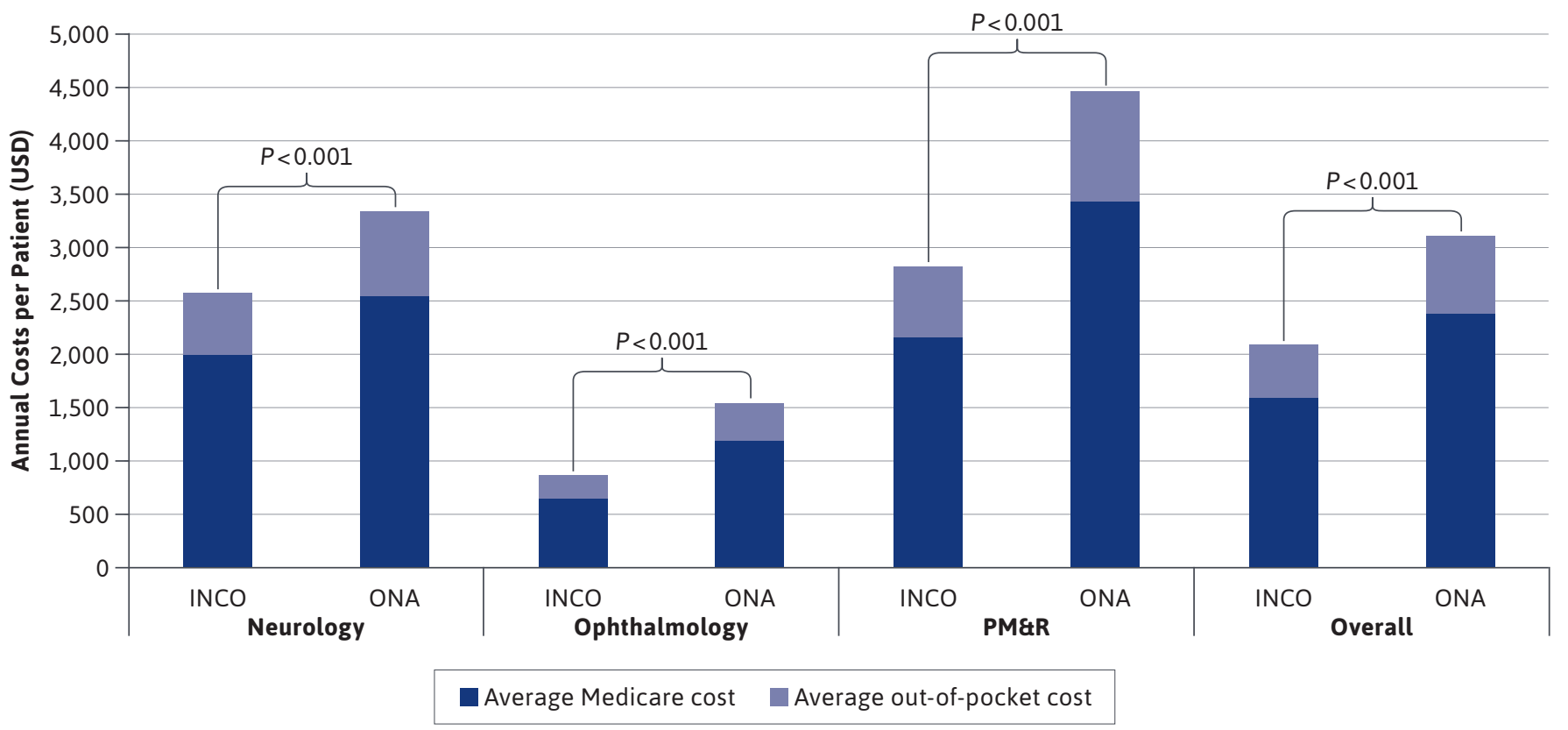

INCO = incobotulinumtoxin $A ; O N A=$ onabotulinumtoxin $A ; P M \& R=$ physical medicine and rehabilitation; USD=U.S. dollar

for incobotulinumtoxinA versus onabotulinumtoxinA across all scenarios (all with $\mathrm{P}<0.001)$ as well: neurology $(\$ 2,005$ vs. \$2,558), ophthalmology (\$679 vs. \$1,193), physical medicine and rehabilitation (\$2,154 vs. \$3,437), and overall $(\$ 1,613$ vs. \$2,396). Finally, OOP cost PPPY for incobotulinumtoxinA versus onabotulinumtoxinA was also significantly less for all scenarios assessed (all with $\mathrm{P}<0.001)$ : neurology (\$579 vs. \$772), ophthalmology (\$205 vs. \$356), physical medicine and rehabilitation (\$675 vs. \$1,028), and overall (\$486 vs. \$719). The most efficient providers were more likely to use incobotulinumtoxinA than the least efficient (Figure 3). The reported Medicare wastage rate was also higher for onabotulinumtoxinA (7.83\%) than for incobotulinumtoxinA (4.77\%).

IncobotulinumtoxinA had similar dosing for all 3 specialties, with an overall ratio of 0.94:1 versus onabotulinumtoxinA. Injections PPPY were significantly lower overall for incobotulinumtoxinA versus onabotulinumtoxinA (2.29 vs. 2.42; $\mathrm{P}=0.02)$. However, this phenomenon was not seen across all specialties (Table 2).

\section{OTHER PRACTICE TRENDS}

Practice patterns varied among specialties. Ophthalmologists treated almost exclusively blepharospasm (100\% of physicians) with a small amount of cervical dystonia (0.5\%) as well. Physical medicine and rehabilitation treated predominantly spasticity (90.5\% of physicians) followed by cervical dystonia (27.2\%) and blepharospasm (4.1\%). Neurology was more mixed, treating cervical dystonia (83.7\%), blepharospasm (51.5\%), spasticity (45.3\%) and sialorrhea (11.1\%).

\section{BUDGET IMPACT ANALYSIS}

Baseline total BoNT spend in 2018 was \$382 million. Market shares from that year were used as the base-case scenario, with incobotulinumtoxinA representing a 5.2\% overall market share versus onabotulinumtoxinA. Total BoNT spend from the physicians in this analysis totaled \$70.9 million, or $18.6 \%$ of total BoNT spend. Total BoNT annual spend by Medicare Part B in the base-case scenario is projected to reach $\$ 505$ million by 2023, with a 3-year total of $\$ 1.43$ billion. Projected cost savings in BoNT spend over the 3-year time horizon totaled $\$ 32.9$ million, for a $20 \%$ incobotulinumtoxinA market share (\$1.402 billion) versus the base-case scenario (\$1.435 billion). Average annual cost savings were projected to be $\$ 11.0$ million from 2021 to 2023. A sensitivity analysis showed 3-year savings could vary from \$10.4 million to $\$ 55.3$ million across market shares of $10 \%-30 \%$, respectively, when compared with the base-case scenario. 


\section{FIGURE 3 Drug Choice of Bottom and Top Decile of Physicians by Annual Cost per Patient $^{\mathrm{a}}$}
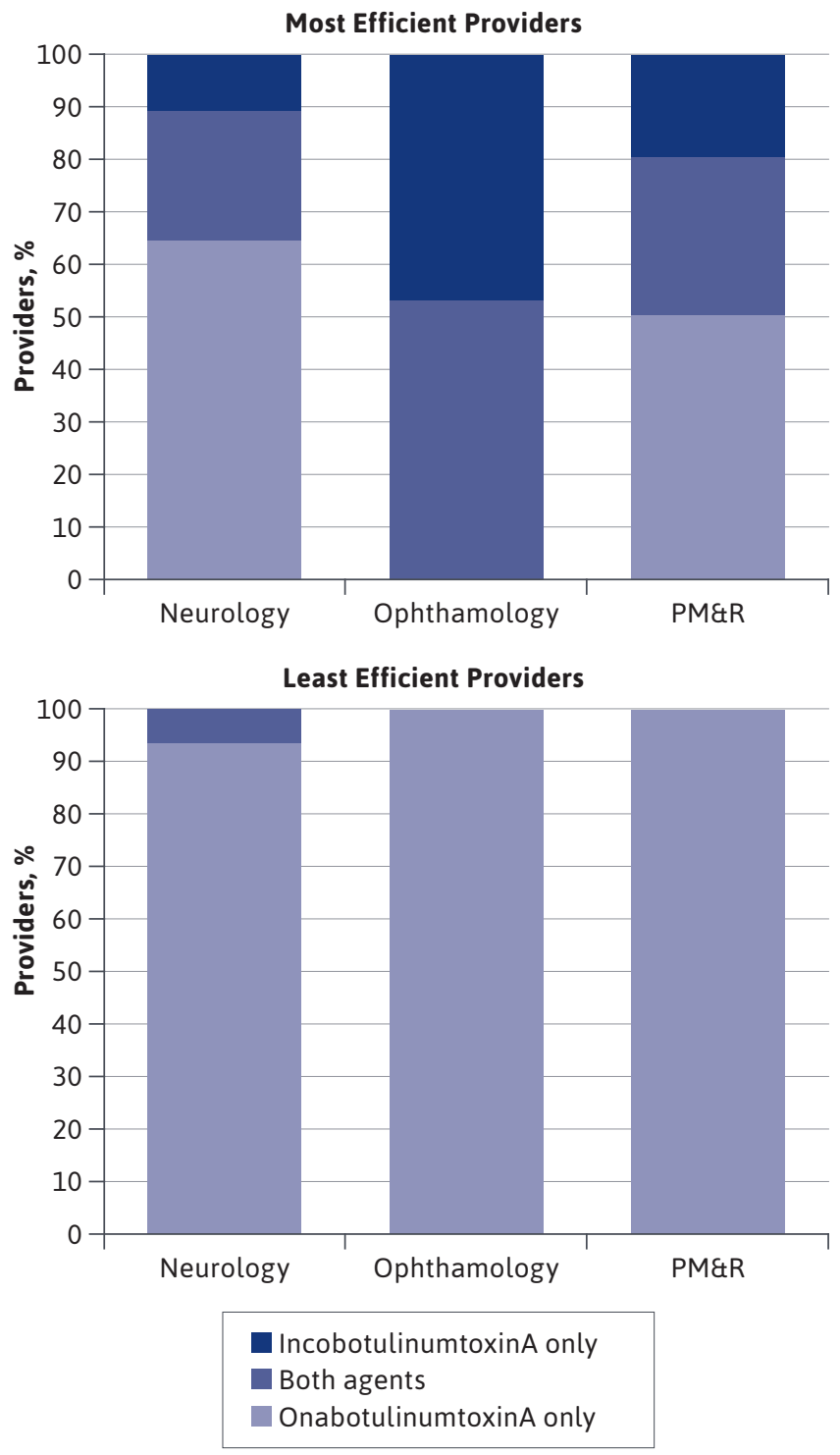

${ }^{a}$ Among physicians with $\geq 20$ BoNT patients.

BoNT = botulinum toxin; $P M \& R=$ physical medicine and rehabilitation .

\section{Discussion}

The present analysis showed in a large Medicare population that incobotulinumtoxinA was lower in cost and reduced wastage compared with other BoNT agents in the therapeutic area of movement disorder treatment. To the author's knowledge, this represents the largest BoNT claims analysis to date of approximately 34 million lives, representing the estimated $70 \%$ of all 53.4 million Medicare Part B beneficiaries who are not enrolled in Medicare Advantage. ${ }^{1,13}$ Additionally, the budget impact analysis estimates that even modest increases in adoption rates of incobotulinumtoxinA for its FDA-approved indications within the BoNT drug class would lead to material cost savings for the federal government and taxpayers via the Medicare system. Although this analysis focused on the BoNT drug class, the authors hope this analysis fuels a broader discussion about pursuing policies within CMS to promote cost-effective care in the Part B space.

The results of this analysis align with previously published results of a BoNT payer analysis of 15 million lives.12 The present analysis found similar numbers of injections PPPY. Additionally, Eckwright et al. (2019) showed an average dose conversion rate per claim for incobotulinumtoxin A:onabotulinumtoxinA ranging from 0.8:1 to 1:1 depending on indication, which aligns with the present findings. That analysis also showed incobotulinumtoxinA to be a less costly therapeutic option versus onabotulinumtoxinA in a commercially insured cohort of patients with a similar time between injections. The present analysis confirms those outcomes in approximately 3 times the patient population and 5 times the number of BoNT injection claims. Although the previous analysis did not discuss wastage in depth, it also showed the largest relative savings for the blepharospasm indication, which aligns with the present findings.

Several policy proposals for Medicare Part B have been discussed over the past 20 years. There was a 2016 policy proposal to vastly change reimbursement methods to "improve quality of care and deliver better value for Medicare beneficiaries." Among other recommended changes, the policies first phase would have changed reimbursement of drugs to ASP plus $2.5 \%$ plus a flat fee. ${ }^{14}$ The second phase would have implemented value-based purchasing similar to commercial pharmacy benefits management organizations. ${ }^{14}$ In the end this policy never took hold.

The Competitive Acquisition Program (CAP), which was operational from 2006 through 2008, was a voluntary program that aimed to separate physicians from the purchasing and billing of medications provided in the physician's office..$^{15,16}$ The program included 180 selected drugs and biologics to be distributed via private vendor specialty pharmacy(s), which would be chosen through a competitive bidding process. ${ }^{16}$ However, multiple issues including having no discernible cost savings, provider dissatisfaction, 


\section{TABLE 2 Dose and Injection Outcomes}

\begin{tabular}{|c|c|c|c|c|c|c|c|c|c|c|c|c|}
\hline & \multicolumn{3}{|c|}{ Neurology } & \multicolumn{3}{|c|}{ Ophthalmology } & \multicolumn{3}{|c|}{ PM\&R } & \multicolumn{3}{|c|}{ Totals } \\
\hline & Ona & Inco & $P$ value & Ona & Inco & $P$ value & Ona & Inco & $P$ value & Ona & Inco & $P$ value \\
\hline Physicians, n & 322 & 53 & - & 378 & 42 & - & 137 & 17 & - & 837 & 112 & - \\
\hline Unique patients, $\mathrm{n}$ & 14,157 & 1,390 & - & 8,673 & 756 & - & 4,611 & 367 & - & 27,441 & 2,513 & - \\
\hline Unique BoNT visits, $\mathrm{n}$ & 35,658 & 3,298 & - & 22,037 & 1,827 & - & 10,784 & 742 & - & 68,479 & 5,867 & - \\
\hline Average dose, units, $\mathrm{n}$ & 213 & 216 & 0.77 & 73 & 72 & 0.71 & 306 & 268 & 0.17 & 197 & 185 & 0.06 \\
\hline Dose ratio & 1 & 1.01 & - & 1 & 0.98 & - & 1 & 0.88 & - & 1 & 0.94 & - \\
\hline Injections per patient/year & 2.42 & 2.30 & 0.10 & 2.49 & 2.40 & 0.33 & 2.26 & 1.99 & $<0.001^{a}$ & 2.42 & 2.29 & $0.02^{\mathrm{a}}$ \\
\hline
\end{tabular}

aStatistically significant.

INCO = incobotulinumtoxin $;$; ONA = onabotulinumtoxin $;$ BONT= botulinum toxin; $P M \& R=$ physical medicine and rehabilitation

physician attrition, and low vendor participation in the program led to the suspension of CAP by the end of $2008 .{ }^{16}$ A new model has been proposed by the U.S. Department of Health and Human Services (HHS), with several key differences from the original CAP program. Differences of the new proposal include that physician participation would be mandatory, reimbursement would be linked to an international pricing index that would likely be less than ASP, and physician groups would be eligible vendors. ${ }^{16}$

There have been other discussions about moving Medicare Part B drugs over to Medicare Part D for a similar and managed formulary process. In May 2018, HHS made this proposal, along with a series of others, as part of a plan to lower drug prices. ${ }^{17,18}$ An analysis of this proposal found that drug spend could be reduced by $7 \%$ to $18 \%$ after rebates..$^{19}$ It did also find that OOP costs may go up for some beneficiaries. ${ }^{19}$ Costs and consequences of such proposals need to be considered at both the patient and system levels.

Medicare Part B recently took the important step of tracking wastage starting in 2017 by requiring a JW modifier on claims that specifies the amount of discarded drugs or biologics. ${ }^{20}$ This policy change requires that this wastage be documented in the patient's medical records as well. ${ }^{20}$ However, there are currently no financial or policy incentives to deter waste because Medicare still pays in full for discarded drug. ${ }^{20}$ OnabotulinumtoxinA had increased rates of wastage versus incobotulinumtoxinA per CMS data. The availability of a 50-unit vial with incobotulinumtoxinA provides an opportunity to potentially reduce cost within this drug class in addition to cost savings attained on a per-unit basis. The authors of the present analysis suggest that Medicare Part B consider a policy assessing and recommending the most efficient vial size and/or that the drug with the most efficient vial size be used. These may be decisions that would need to be made on a drug class by class, and even indication by indication. However, such policies may lead to an outsize return on investment of implementation.

The idea of having clinicians being stewards of highvalue, data-driven, cost-conscious care is a concept that has circulated for some time. ${ }^{21}$ Because health care costs have been increasing unsustainably, this concept emphasizes the provider role in containing costs while maintaining quality of care. Data have shown that medical students are more likely than physicians to believe that cost to society is an important factor in treatment decisions. ${ }^{22}$ This shows a potential disconnect between physician perspectives before entering practice and constraints of real-world practice. Alignment of incentives for cost and clinical outcomes is essential for both clinician and payer to benefit in the most cost-effective scenarios. Such incentives in the current U.S. health care systems are not well aligned, which likely plays a large role in the underuse of available costeffective agents including within the BoNT space.

OnabotulinumtoxinA, unlike incobotulinumtoxinA, has a wider array of therapeutic FDA indications extending beyond the movement disorder space and into other clinical specialties such as urology, dermatology, and migraine. Such circumstances can be more complicated from a formulary management standpoint. Payers do often have different formulary policies for medications based on different respective indications. However, it can prevent a more simplistic approach of having a single formulary agent across all indications. Similarly, at the health system level, different institutions must decide whether to have a simple overarching formulary policy for a single agent versus 
having a more nuanced approach that varies by indication or specialty. These health system level practicalities should not be discounted, as the ability to develop and maintain more nuanced formulary policies may depend on the formulary and administrative pharmacist resources of each given institution.

\section{LIMITATIONS}

There are several limitations to this analysis. First, this is a retrospective analysis of aggregated Medicare claims data of elderly patients in the United States.

Second, the Medicare data available on the CMS website and used in the analysis excludes Medicare Advantage beneficiaries who account for approximately $30 \%$ of the overall Medicare population. ${ }^{1}$

Third, injections per year were taken from a calendar year of data. Therefore, not every patient in the analysis was necessarily observed for an entire year.

Fourth, per-claim wastage data were not available in the dataset used for this analysis. Therefore, average wastage rate for each respective drug from the most recent reported Medicare wastage rates were added on to the costs for each drug for most scenarios. Future studies should assess BoNT wastage by specialty and indication specifically.

Fifth, the publicly available Medicare data file used for this analysis only provides data on J codes and CPT-4 codes in which the provider had $\geq 11$ unique patients. Data on physicians who inject only a small number of BoNT patients were not available and, therefore, were not assessed. Additionally, this meant for physicians included in the analysis who had low volumes (<11 patients) for a given CPT- 4 or J code, these data were also not available for inclusion.

Sixth, the potency units of incobotulinumtoxinA are specific to the preparation and assay method used. They are not interchangeable with onabotulinumtoxinA and, therefore, units of incobotulinumtoxinA cannot be compared with or converted into units of onabotulinumtoxinA.

Seventh, the data used for this analysis were from the most recent calendar year available. However, market share may have already changed among the agents. IncobotulinumtoxinA annual use in Medicare Part B grew an average of $24.9 \%$ annually from 2016 to 2018, outpacing onabotulinumtoxinA (8.2\% growth). ${ }^{1}$ Future studies should confirm savings projected from this analysis.

Eighth, it should be noted that this analysis did not represent the entirety of Medicare movement-disorder BoNT spend, given that physicians who did not inject predominantly for movement disorders were excluded from this analysis and lower-volume provider data were unavailable. Conversely, a portion of the BoNT data (estimated to be $12.2 \%$ ) included in this analysis was not movement disorder-related.

Finally, the data presented include analysis of therapeutic uses of incobotulinumtoxinA and onabotulinumtoxinA that may exceed such products' FDA-approved indications and so is not intended to imply claims of safety or efficacy for either product outside of the respective product's FDA indications.

\section{Conclusions}

In this analysis of the Medicare Part B population, incobotulinumtoxinA had reduced cost per patient, reduced patient OOP spending, and lower wastage compared with other BoNT used for movement-disorder treatment. Potential to reduce government and overall spend on Medicare Part B use of BoNT agents via implementation of cost-effectiveness evaluations holds potential for bolstering evidence-based use of taxpayer funds, both within the BoNT drug class and for Medicare Part B on a broader level. Therefore, awareness of the issue is important and further research is warranted in order to identify the best methods for implementing such policies.

\section{DISCLOSURES}

This research received no external funding. Kazerooni was an employee of Merz Pharmaceuticals at the time of the analysis. Watanabe received no compensation or funding for this research project. Watanabe is a member of the National Academies of Sciences, Engineering, and Medicine Forum on Drug, Discovery, Development, and Translation. This information, content, and conclusions are those of the authors and should not be construed as the official position or policy of nor should any endorsements be inferred by the U.S. government or the National Academies of Sciences, Engineering. 


\section{REFERENCES}

1. Centers for Medicare \& Medicaid Services. Medicare Part B drug spending dashboard. December 22, 2020. Accessed January 8, 2021. https://www. cms.gov/Research-Statistics-Data-andSystems/Statistics-Trends-and-Reports/ Information-on-Prescription-Drugs/ MedicarePartB

2. Centers for Medicare \& Medicaid Services. Medicare Part D drug spending dashboard and data. December 22, 2020. Accessed January 8, 2021. https://www. cms.gov/Research-Statistics-Data-andSystems/Statistics-Trends-and-Reports/ Information-on-Prescription-Drugs/ MedicarePartD

3. Kuznar W. ASP plus $6 \%$ will fall by the wayside, but what are the alternatives? Am Health Drug Benefits. 2015;8 (Spec Issue):31.

4. Centers for Medicare \& Medicaid Services. CMS roadmaps overview. Accessed January 8, 2021. https://www. cms.gov/Medicare/Quality-InitiativesPatient-Assessment-Instruments / QualityInitiativesGenInfo/Downloads/ RoadmapOverview_OEA_1-16.pdf

5. Cubanski J, Damico A, Neuman T, Jacobson G; Kaiser Family Foundation. Sources of supplemental coverage among Medicare beneficiaries in 2016. November 28, 2018. Accessed January 8, 2021. https://www. kff.org/medicare/issue-brief/ sources-of-supplemental-coverageamong-medicare-beneficiaries-in-2016/

6. Centers for Medicare \& Medicaid Services. Medicare Part B discarded drug units report. December 22, 2020. Accessed January 8, 2021. https://www. cms.gov/research-statistics-data-sys tems/cms-drug-spending/medicarepart-b-discarded-drug-units-report
7. Benecke R, Jost WH, Kanovsky P, Ruzicka E, Comes G, Grafe S. A new botulinum toxin type A free of complexing proteins for treatment of cervical dystonia. Neurology. 2005;64(11):1949-51.

8. Roggenkämper P, Jost WH, Bihari K, Comes G, Grafe S; NT 201 Blepharospasm Study Team. Efficacy and safety of a new botulinum toxin type A free of complexing proteins in the treatment of blepharospasm. J Neural Transm (Vienna). 2006;113(3):303-12.

9. Xeomin (incobotulinumtoxina injection, powder, lyophilized, for solution). Merz Pharmaceuticals, December 2020. Accessed January 8, 2021. https:// dailymed.nlm.nih.gov/dailymed/fda/ fdaDrugXsl.cfm?setid=ccdc3aae-6e2d4cd0-a51c-8375bfee9458\&type $=$ display

10. Kazerooni R, Broadhead C. Costutility analysis of botulinum toxin type A products for the treatment of cervical dystonia. Am J Health Syst Pharm. 2015;72(4):301-07.

11. Centers for Medicare \& Medicaid Services. Medicare provider utilization and payment data: physician and other supplier. December 3, 2020. Accessed January 8, 2021. https://www.cms. gov/Research-Statistics-Data-andSystems/Statistics-Trends-and-Reports/ Medicare-Provider-Charge-Data/ Physician-and-Other-Supplier

12. Eckwright DJ, Burke JP, Gleason PP. Real-world botulinum toxin utilization and treatment cost for cervical dystonia and limb spasticity among 15 million commercially insured members. Poster presented at: AMCP Nexus; October 29-November 1, 2019; National Harbor, MD. Accessed January 8, 2021. https://www.jmcp.org/ doi/pdf/10.18553/jmcp.2019.25.issue-10-a
13. Centers for Medicare \& Medicaid Services. MDCR ENROLL AB 3. 2012-2017. Accessed January 8, 2021. https://www. cms.gov/Research-Statistics-Data-andSystems/Statistics-Trends-and-Reports/ CMSProgramStatistics/2017/Downloads/ MDCR_ENROLL_AB/2017_CPS_MDCR_ ENROLL_AB_3.pdf

14. U.S. Department of Health and Human Services. Medicare program: Part B drug payment model proposed rule. March 11, 2016. Accessed January 8, 2021. https:// s3.amazonaws.com/public-inspection. federalregister.gov/2016-05459.pdf

15. Lace DA. The competitive acquisition program for drugs and biologicals. Manag Care. 2006;15(7 Suppl 3):21-24.

16. The Commonwealth Fund. Old lessons for the new Medicare Part B drug payment model. November 26, 2018. Accessed January 8, 2021. https://www. commonwealthfund.org/blog/2018/newmedicare-part-b-drug-payment-model

17. U.S. Department of Health and Human Services. American patients first: the Trump administration blueprint to lower drug prices and reduce out-of-pocket costs. May 2018. Accessed January 8, 2021. https://www.hhs.gov/sites/default/files/ AmericanPatientsFirst.pdf

18. U.S. Department of Health and Human Services. Fixing healthcare: driving value through smart purchasing and policy. May 16, 2018. Accessed January 8, 2021. https://www.hhs.gov/about/leadership/ secretary/speeches/2018-speeches/ fixing-healthcare-driving-value-throughsmart-purchasing-and-policy.html

19. Hwang TJ, Jain N, Lauffenburger JC, Vokinger KN, Kesselheim AS. Analysis of proposed Medicare Part B to Part D shift with associated changes in total spending and patient cost-sharing for prescription drugs. JAMA Intern Med. 2019;179(3):37480. doi: 10.1001/jamainternmed.2018.6417 
20. Centers for Medicare \& Medicaid Services. Medicare program JW modifier: drug/biological amount discarded/ not administered to any patient: frequently asked questions. August 26, 2016. Accessed January 8, 2021. https://www.cms.gov/Medicare/ Medicare-Fee-for-Service-Payment/ HospitalOutpatientPPS/Downloads/ JW-Modifier-FAQs.pdf
21. Owens DK, Qaseem A, Chou R, Shekelle P; Clinical Guidelines Committee of the American College of Physicians. High-value, cost-conscious health care: concepts for clinicians to evaluate the benefits, harms, and costs of medical interventions. Ann Intern Med. 2011;154(3):174-80. doi: 10.7326/0003-4819154-3-201102010-00007
22. Leep Hunderfund AN, Dyrbye LN, Starr SR, et al. Attitudes toward costconscious care among U.S. physicians and medical students: analysis of national cross-sectional survey data by age and stage of training. BMC Med Educ. 2018;18(1):275. doi: 10.1186/ s12909-018-1388-7 